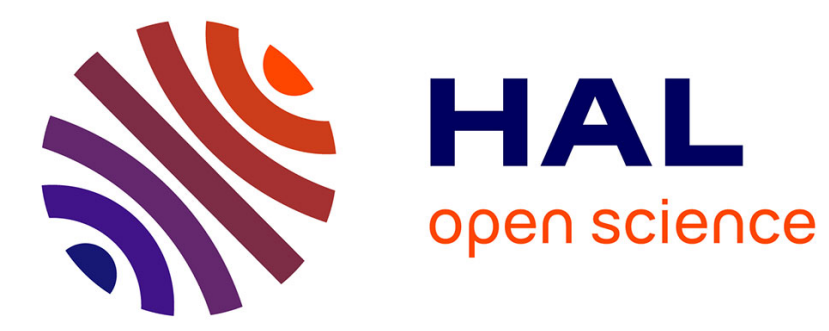

\title{
SPIN PHYSICS IN LEPTO- AND PHOTOPRODUCTION
}

\author{
B. Pire
}

\section{To cite this version:}

B. Pire. SPIN PHYSICS IN LEPTO- AND PHOTOPRODUCTION. Journal de Physique Colloques, 1985, 46 (C2), pp.C2-45-C2-54. 10.1051/jphyscol:1985205 . jpa-00224516

\section{HAL Id: jpa-00224516 https://hal.science/jpa-00224516}

Submitted on 1 Jan 1985

HAL is a multi-disciplinary open access archive for the deposit and dissemination of scientific research documents, whether they are published or not. The documents may come from teaching and research institutions in France or abroad, or from public or private research centers.
L'archive ouverte pluridisciplinaire HAL, est destinée au dépôt et à la diffusion de documents scientifiques de niveau recherche, publiés ou non, émanant des établissements d'enseignement et de recherche français ou étrangers, des laboratoires publics ou privés. 
JOURNAL DE PHYSIQUE

Colloque $\mathrm{C} 2$, supplément au $\mathrm{n}^{\circ} 2$, Tome 46, février 1985

page $\mathrm{C} 2-45$

SPIN PHYSICS IN LEPTO- AND PHOTOPRODUCTION

B. Pire

CERN, CH-1211 Geneva, Switzertand

Résumé - Les prédictions de la chromodynamique quantique relatives aux observables de spin sont passées en revue pour les réactions de leptoproduction et de photoproduction. L'étude expérimentale des différentes asymétries devrait clarifier notre compréhension de la structure fine de la matière. Elle constitue en tous cas une série de tests qu'on ne peut se dispenser d'appliquer à la théorie des interactions fortes.

Abstract - QCD predictions for spin observables in leptoproduction and photoproduction are reviewed. The experimental study of various spin asymmetries should clarify our understanding of the deep structure of matter. It will constitute a set of tests that the candidate theory of strong interactions has to pass.

\section{INTRODUCTION}

Leptoproduction processes constitute the building block of our understanding of the deep structure of matter. Its significance has been experimentally established by the crucial SLAC experiments which revealed the partonic structure of the proton. Theoretically, the by-now classical operator product expansion analysis showed that deep inelastic cross-sections could be factorized as a long-distance part convoluted with a hard subprocess which can be described as a partonic reaction. Considering spin-dependent quantities does not change these conclusions. The theoretical framework and many predictions have thus been developed in the last decade /1/. Here we must again emphasize the importance of measuring these observables. Indeed, the spin structure of the underlying theory is crucial. For instance, the vector nature of the gluon field in QCD, although it may be inferred from unpolarized measurements, has to be "directly" seen in polarized experiments. For definiteness, I will stick to electroproduction, having in mind the future HERA experiments. Neutrino experiments can be analysed along the same lines, with some obvions changes.

Hard photoproduction processes, that is, large $p_{T}$ jet production and heavy flavour creation, share many of the advantageous features of leptoproduction. The successful photoproduction experiments now running at CERN and FNAL might easily be upgraded to a polarized version. They would allow the testing of the various QCD predictions that we review below.

\section{I - POLARIZED LEPTOPRODUCTION}

Since the discovery of the partonic structure of the proton in the celebrated SLAC electroproduction experiments, leptoproduction processes have been much studied theoretically. In the polarized case too, they are at the forefront of the study of the deep structure of hadrons. 


\section{1 - The Polarized Structure Functions}

are the basic quantities to study. Let us briefly recall some basic definitions (see Fig. 1). Restricting ourselves to longitudinal polarization, and with obvious notations (lepton energies $E$ and $E^{\prime}$, and scattering angle $\theta$ being measured in the laboratory frame), we have

$$
\frac{d^{2} \sigma_{++}}{d q^{2} d E^{\prime}}-\frac{d^{2} \sigma_{+}-}{d q^{2} d E^{\prime}}=\frac{4 \pi \alpha^{2}}{M\left(E-E^{\prime}\right) E^{2} q^{2}}\left\{\left(E_{+} E^{\prime} \cos \theta\right) G_{1}+\frac{q^{2} M G}{p \cdot q}\right\}
$$

where the second term in the bracket dies off at high energy.

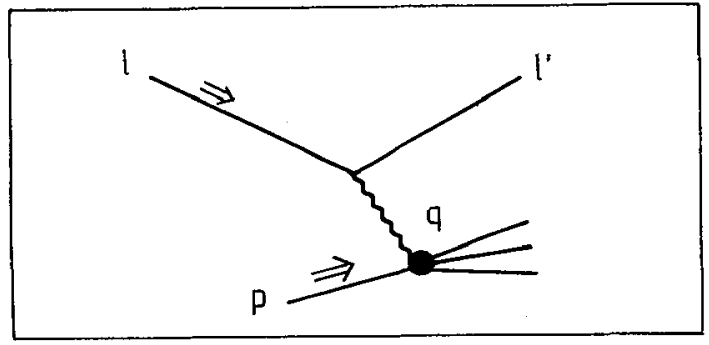

Fig. 1 - Inclusive leptoproduction kinematics.

In the parton model the polarized structure function $G_{1}$ shows a scaling behaviour:

$$
G_{1}\left(p \cdot q, q^{2}\right) \longrightarrow G_{1}\left(x=-q^{2} / 2 p \cdot q\right)
$$

It is related to the helicity distribution functions in a proton,

$$
\Delta q(x)=q_{++}(x)-q_{-+}(x)
$$

where the subscripts denote the parton and proton helicity states, through the relation:

$$
G_{1}(x)=\frac{1}{2} \sum_{q} e_{q}^{2} \Delta q(x)
$$

Current algebra techniques lead to a very important sum rule $/ 2 /$

$$
\int_{0}^{1} d x\{\Delta u(x)-\Delta d(x)+\Delta \bar{u}(x)-\Delta \bar{d}(x)\}=\frac{G_{A}}{G_{V}}=1.25
$$

This scaling behaviour is logarithmically broken in an asymptotically free theory like QCD. The renormalization group equations allow us to calculate this $Q^{2}$ dependence perturbatively. Evolution equations may be written in a similar way as for the unpolarized case

$$
\Delta q_{a}\left(x, Q^{2}\right)=\Delta q_{a}\left(x, Q_{0}^{2}\right)+\int_{Q_{0}^{2}}^{Q^{2}} \frac{d q^{2}}{q^{2}} \frac{\alpha_{s}\left(q^{2}\right)}{2 \pi} \int_{x}^{1} \frac{d z}{z} \Delta P_{a b}(z) \Delta q_{b}\left(\frac{x}{z}, q^{2}\right)
$$

The helicity-dependent parton branching probabilities $\Delta \mathrm{P}_{a b}(z)$ can be written (for three flavours) as: 


$$
\begin{aligned}
& \Delta P_{q q}(z)=\frac{4}{3}\left(\frac{1+z^{2}}{1-z}\right)_{+} \\
& \Delta P_{q g}(z)=\frac{1}{2}\left(z^{2}-(1-z)^{2}\right) \\
& \Delta P_{g q}(z)=\frac{4}{3}(2-z) \\
& \Delta P_{g g}(z)=3\left\{\frac{1+z^{4}}{z}-\frac{(1-z)^{3}}{z}+\left(\frac{1+z^{4}}{1-z}\right)_{+}\right\}+\frac{9}{2} \delta(1-z)
\end{aligned}
$$

They display the remarkable properties that

$$
\Delta P_{a b}(z) / P_{a b}(z) \rightarrow 1 \text { when } z \rightarrow 1
$$

(Fast debris remember the helicity of their parents)

$$
\Delta P_{9 q}(z) / P_{q q}(z)=1 \text { for all } z
$$

(Helicity conservation along the fermion line).

The Bjorken sum rule gets some $Q^{2}$ violation:

$$
\int_{0}^{1} d x\left\{\Delta u\left(x, Q^{2}\right)-\Delta d\left(x, Q^{2}\right)+\Delta \bar{u}\left(x, Q^{2}\right)-\Delta \bar{d}\left(x, Q^{2}\right)=\frac{G_{A}}{G_{r}}\left(1-\frac{\alpha_{s}\left(Q^{2}\right)}{\pi}\right)\right.
$$

It is important to note that none of the $\Delta \mathrm{P}_{a b}(z)$ vanish identically. Consequently, a statement such as "sea quarks are unpolarized" is contradictory to QCD; were it true at $Q_{0}^{2}$, it would become wrong at $Q_{1}^{2}$. Experimental data thus leads us to two very interesting and distinctive points.

a) The polarized structure function at some $Q_{0}^{2}$ gives us information about the longdistance behaviour of quark and gluon interactions inside a hadron. Theoretically, this problem seems very difficult and needs some powerful non-perturbative method still to be discovered. Not much progress has been achieved in this direction recently, and we are still at the level of clever, but, however, quite naive, models $/ 3,4 \%$. Since it bes.t fits avallable experimental data, let us briefly recall the model of Carlitz and Kaur /3/. A spin dilution factor $\delta(x)$ is introduced as:

$$
\delta(x)=\left[1+\lambda \frac{(1-x)^{2}}{\sqrt{x}}\right]^{-1}
$$

such as $\delta(1)=1$ and $\delta(0)=0$. The $u$ and $d$ quark helicity distribution functions are written as:

$$
\begin{aligned}
& \Delta u(x)=\delta(x)\left\{u_{v}(x)-\frac{2}{3} d_{v}(x)\right\} \\
& \Delta d(x)=-\delta(x) d_{v}(x) / 3
\end{aligned}
$$

In this framework, a recent analysis $/ 5 /$ finds that at $Q_{0}^{2}=5 \mathrm{GeV}^{2}, 73 \%$ of the proton spin is carried by valence quarks, the remaining $27 \%$ being taken away by sea quarks and gluons. The few available experimental data are well accounted for by such a model. Figure 2 shows the quark asymmetry: 


$$
A_{q}(x)=\frac{\sum_{q} e_{q}^{2} \Delta_{q}(x)}{\sum_{q} e_{q}^{2} q(x)}
$$

as measured for $Q^{2}=2-10 \mathrm{GeV}^{2}$ by the SLAC-Yale collaboration in electroproducion $/ 6 /$ together with the model prediction $/ 5 /$.

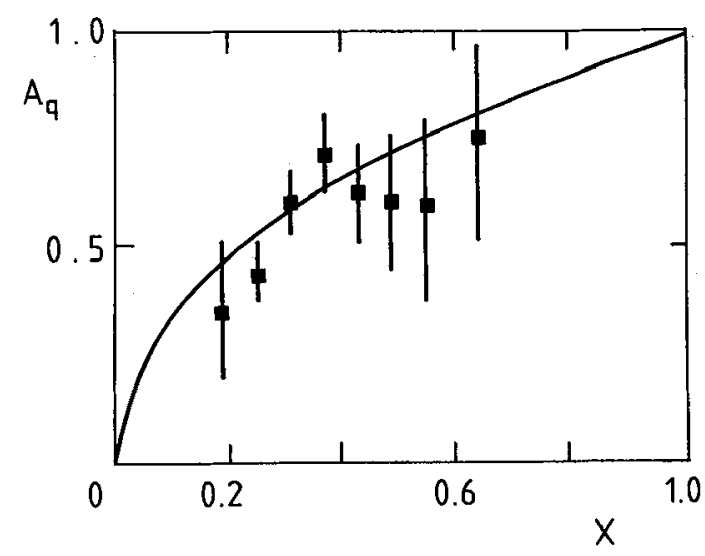

Fig. 2 - The quark asymmetry in electroproduction.

These data, however, test mainly the valence part and do not give much insight into the very interesting gluon part. Speculations on this latter may lead to very different expectations, as seen in Fig. 3, where the gluon asymmetry

$$
A_{g}(x)=\Delta g(x) / g(x)
$$

is shown for two models, $A / 4 /$ and $B / 7 /$.

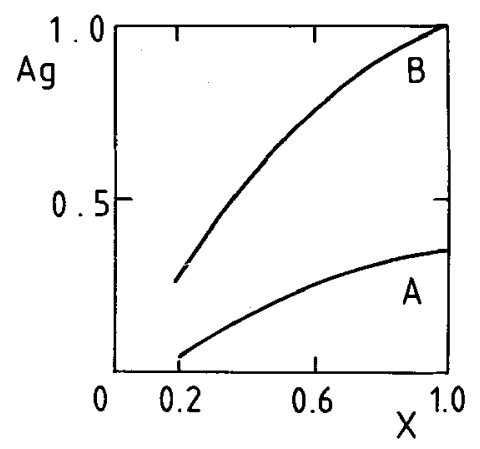

Fig. 3 - The gluon asymmetry in two models

Note also that it would be interesting to get polarized neutrons to study the helicity distribution function of $u$ quarks in neutrons [which is $d\left(x, Q^{2}\right)$ ]. Indeed, 
the Bjorken sum rule supplemented by some natural (but still speculative) assumptions predicts a quite distinctive $x$ behaviour of this function $/ 8 /$.

b) The evolution with $Q^{2}$ tests the short-distance part, calculated in perturbation theory. Detalled predictions and parametrizations have been worked out /3-5/ using different techniques for solving the evolution equations written above. In the gluon case, the $Q^{2}$ dependence turns out to be quite dramatic, as shown in Fig. 4 , where the parametrization of Ref. 5 has been used. The reason is that the nonAbelian triple gluon coupling makes the first moment of the polarized gluon distribution function linear in $\ell n Q^{2} / 9 /$ An experimental confirmation of such a behaviour would be very welcome.

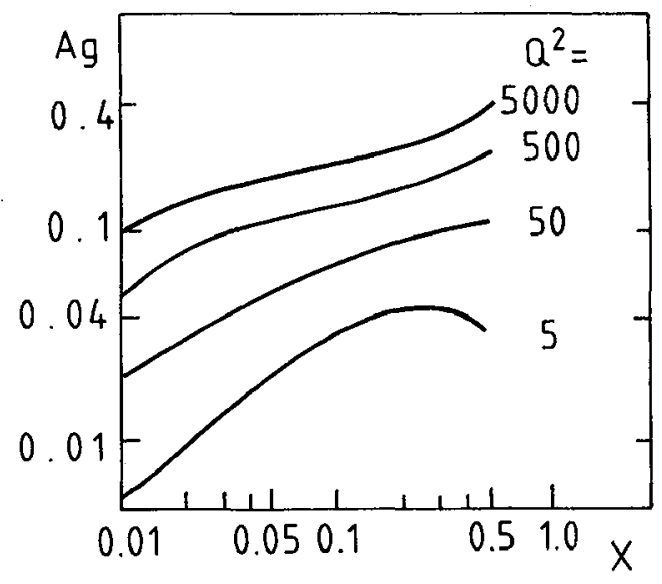

Fig. 4 - The variation with $Q^{2}$ of the gluon asymmetry.

It turns out, however, that it is quite difficult to extract the gluon distribution function from leptoproduction data. We shall see below that this can be done using other experimental set-ups.

\section{2 - And When Only One Initial Particle is Polarized ....}

you can still get a lot of physics results. Two examples are:

a) the inclusive production of a polarized hadron when the target only is polarized. This process $/ 10 /$, shown in Fig. 5, allows the study of polarized fragmentation functions, a non-perturbative quantity about which one may speculate /11/, but not yet predict, and of its $Q^{2}$ dependence which is governed by evolution equations similar to those for distribution functions. 


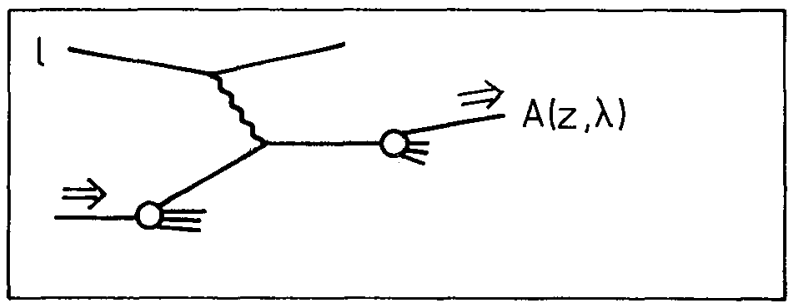

Fig. 5 - Leptoproduction of a polarized hadron.

The observed hadron might be vector mesons or baryons, $\Lambda$ 's being particularly suitable.

b) The observation of T-odd azimuthal asymmetry when only the lepton beam is polarized /12/. Let us consider large $\mathrm{p}_{\mathrm{T}}$ jet leptoproduction. At lowest order in QCD it is described by the Born graphs of Fig. 6a. Defining the azimuth $\phi$ as the angle of the leptonic plane (defined by $\vec{l}$ and $\vec{l} \cdot$ ) and of the scattering plane (defined by $\vec{k}$ and $\vec{r}$ ), it is easy to show that there is no sin $\phi$ term in the differential cross-section at that order. Such a T-odd contribution arises when loop graphs involving an absorptive part are taken into account. Some of these graphs are drawn in Fig. 6b. Their interference with the Born term yields a left-right azimutha1 asymmetry. Note that such an asymmetry vanishes kinematically when $Q^{2} \rightarrow 0$. $0 f$ course, to observe it, one has to tag, say, the quark jet with respect to the gluon jet, which is not very easy but may be done statistically. The calculated asymmetry, which is of $O\left(\alpha_{s}\right)$, turns out to be quite smal1, of the order of $1 \%$. Such statement is very interesting to verify, since it tests our understanding of final state interactions at the perturbative level.

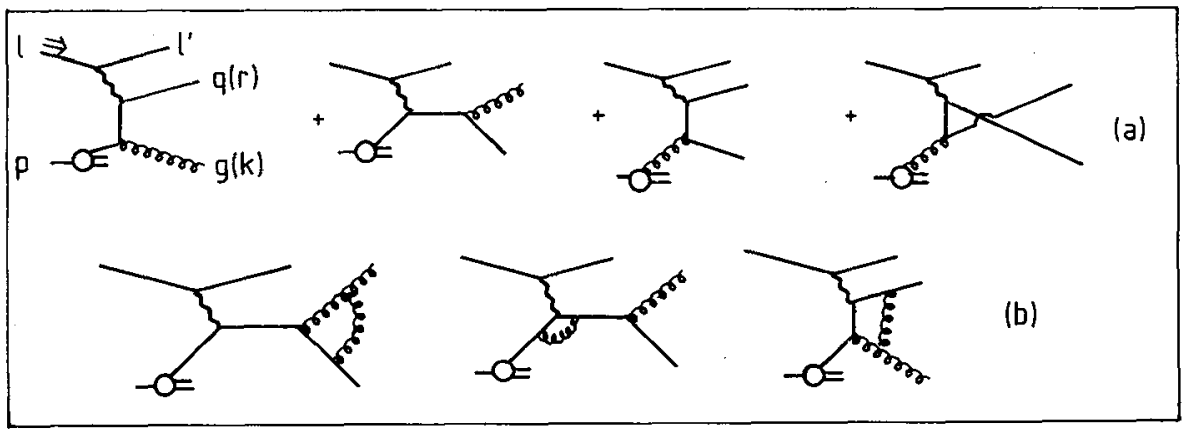

Fig. 6 - Leptoproduction of large $p_{T}$ jets: (a) Born term; (b) some loop diagrams inducing the $\mathrm{T}$-odd azimuthal asymmetry. 
II $-\gamma^{*} \gamma$ PROCESSES

These processes attainable in $\mathrm{e}^{+} e^{-}$and in $\gamma \mathrm{e}^{-}$collisions present some very interesting peculiarities. The pointlike nature of the (quasi) real photon target leads to some simplification in the theoretical treatment, but this has to be recompensed by smaller cross-sections. Dedicated second-generation experiments at the electronpositron colliders or with laser beams might give us some clues about them in the not-too-distant future.

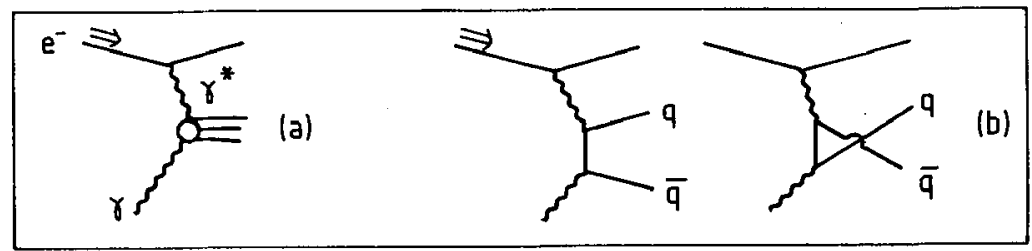

Fig. $7-\gamma^{*} \gamma$ processes: (a) inclusive, (b) jet production.

1 - The Photon Structure Function (Fig. 7a)

Deep inelastic scattering of a polarized lepton on a quasi-real photon can be studied along the same lines as on a hadron. The pointlike nature of the quarkphoton coupling leads to a dramatic Improvement: the structure functions are perturbatively calculable at leading logarithmic order $/ 13 /$. The non-spin flip function $H_{4}\left(x, Q^{2}\right)$ grows proportionally to $\log Q^{2}$, as does the unpolarized function $G_{1}\left(x, Q^{2}\right)$. It has, however, been recently recognized that at moderate $Q^{2}$, one could not trust this leading order estimate without damage, especially at low $x / 14 /$. The hadronic nature of the photon at low $Q_{0}^{2}$ mixes as a boundary condition with 1 ts pointlike structure expressed by the evolution equations. Experimental facts would be very welcome.

2 - T-odd Effects in the Single Spin Case

Let us consider jet production (FIg. 7b) in polarized leptoproduction on an unpolarlized photon. As discussed above in the hadronic target case, absorptive parts of loop diagrams induce a T-odd azimuthal asymmetry of order $\alpha_{s}{ }^{*}$ The perturbative QCD calculation $115 /$ leads to a $10 \%$ effect, which should be observable in a dedicated experiment.

\section{III - HARD PROCESSES IN REAL PHOTOPRODUCTION}

Real photon beams with high luminosity and energy have been available for some time at CERN and FNAL. They are now at the stage of giving detalled information about hard processes such as large transverse momentum jet production. Longitudinally polarized photon beams are also avallable. The study of single spin and spin-spin effects in these reactions is thus within reach. It turns out that in a number of cases perturbative QCD predicts quite large asymmetries.

\section{1 - Polarized Photon - Unpolarized Target}

Various quantities can be considered. Firstly, the dependence on the angle $\phi$ between the scattering plane and the polarization vector of the initial photon may be written, at the subprocess leve1, as

$$
d \sigma\left(\gamma \gamma_{q} \rightarrow g q\right)=d \sigma(\gamma q \rightarrow g q) \cdot\left[1+\lambda P_{q} \cos 2 \phi\right]
$$




$$
d \sigma\left(\gamma^{\top} g \rightarrow q \bar{q}\right)=d \sigma(\gamma g \rightarrow q \bar{q})\left[1+\lambda P_{g} \cos 2 \phi\right]
$$

where $\lambda$ measures the initial helicity and $P_{g}$ and $P_{g}$ are $0\left(\alpha_{s}\right)$ quantities that may be calculated $/ 16 /$. Differences of the order of $10 \%$ are to be expected. Analogous helicity dependences are also present in deep Compton scattering /17/ where, however, cross-sections may be too sma11 to allow a sensitive test at the $10 \% 1$ evel.

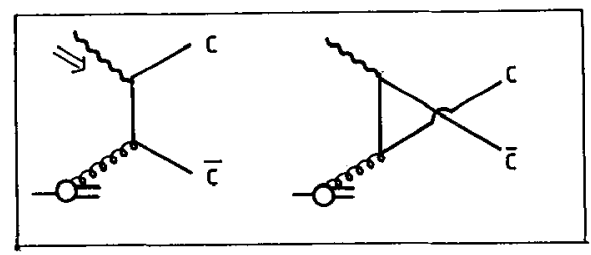

Fig. 8 - The fusion diagram for charm photoproduction.

The case of charm photoproduction has to be considered separately. Indeed, the fact that the charm quark is massive lets us belfeve that its production can be calculated in perturbation theory even at zero momentum transfer. Moreover, it yields a non-zero Born term contribution to the asymmetry. This asymetry vanishes at $p_{T}=0$ for kinematical reasons; integrated over $P_{T}$, its estimated magnitude is about $30 \% / 18 /$.

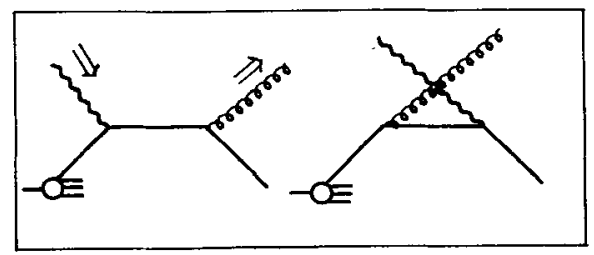

Fig. 9 - The QCD Compton process.

Thirdly, the polarization of the photon is efficiently transmitted to the produced gluon in the QCD Compton graph of FIg. 9. Indeed, denoting by $\phi$ ( $\phi^{\prime}$ ) the angle between the scattering plane and the polarization vector of the photon (gluon), one obtains the Born term level for the subprocess cross-section

$$
d \sigma / d t \sim\left[(-s / t-t / s-2)+4 \cos ^{2}\left(\phi^{\prime}-\phi\right)\right] / s^{2}
$$

where $s$ and $t$ are the usual Mandelstam variables. The first term in the bracket is small near $90^{\circ}$, while the second leads to a strong correlation between the directions of the photon and gluon polarizations $/ 19 /$. The observation of this gluon polarization would be a beautiful manifestation of the vector nature of the gluon. Various experimental signatures have been proposed: the study of the shape of this gluon jet $/ 20 /$ or of the mean transverse momentum out of the scattering plane should signal the polarization of the parent parton. Using models for helicity fragmentation functions, Anselmino and Kroll /21/ have studied in detail the vector meson spin density matrix when this gluon jet fragments in a fast $\rho$ meson. Remember that 
in the meson rest frame, the angular distribution of the $\pi^{+}$depends on the density matrix elements as:

$$
\begin{aligned}
& W(\theta, \phi)=1-\left(\rho_{00}-\frac{\rho_{11}+\rho_{-1-1}}{2}\right)\left(1-3 \cos ^{2} \theta\right) \\
& \quad-3 \sqrt{2} \sin \theta \cos \theta \sin \phi \operatorname{Re}\left(\rho_{10}-\rho_{-10}\right)-3 \sin ^{2} \theta \cos 2 \phi \operatorname{Re} \rho_{1-1}
\end{aligned}
$$

Since vector mesons are known to be produced at quite an extensive rate in large transverse momentum processes, the measurement of these matrix elements seems very feasible.

\section{2 - Polarized Photon - Polarized Target Asymmetries}

The subprocess asymmetry

$$
\hat{A}=\frac{d \sigma_{++} / d t-d \sigma_{+-1} / d t}{d \sigma_{++} / d t+d \sigma_{+-} / d t}
$$

where indices denote the helicities of incoming photons and quarks or gluons, take at lowest order in perturbative QCD the values

$$
\hat{A}_{c}=\frac{s^{2}-t^{2}}{s^{2}+t^{2}}
$$

for the Compton graphs of Fig. 9, and

$$
\hat{A}_{F}=-1
$$

for the fusion graphs of Fig. 8 (with massless quarks). The fact that the Compton graph contribution can be separated by taking differences of $\pi^{+}$and $\pi^{-}$photoproduction yields definite predictions for the related asymmetry which amounts to about $50 \%$ at moderate $\mathrm{p}_{\mathrm{T}} / 22 /$. Separating the Fusion subprocess contribution should lead to a measurement of the gluon helicity distribution function and of its spectacular $Q^{2}$ dependence (in these processes $Q^{2}$ does not denote the virtualness of the photon, but is related to the transverse momentum of the produced jet). The contributions due to the indirect coupling of the photon (through its constituents, the distribution functions of which have been briefly discussed in Section II) have been recently examined $/ 23 /$. Asymmetries in charmonium and charmed particle production have also been discussed $124 /$; the subprocess at work being mostly the fusion graph of Fig. 8, they test the gluon helicity properties. The predictions, however, depend quite a lot on the hadronization model.

For all these asymmetries, next-to-leading order predictions have not yet been calculated. Guided by related calculations, in the Drel1-Yan case for instance /25/, one might guess that such corrections will be small: the reason is that most of the correction factorizes and thus cancels in ratios like asymmetries. This must, however, be checked for each specific reaction.

\section{IV - CONCLUSIONS}

In conclusion, let us stress again that polarized leptoproduction and photoproduction experiments, although difficult, are feasible and should be very fruitful in the near future. They are of great interest theoretically since they will:

- test perturbative QCD predictions for the short-distance part of strong interactions. QCD is believed to be the theory of strong interactions; we cannot dispense with testing its spin predictions;

- yield some important information on the long-distance spin structure of hadrons which we should ultimately be able to compute In QCD by non-perturbative methods. 


\section{REFERENCES}

/1/ For a recent and extensive review of spin physics at short distances, see: CRAIGIE N. et al., Phys. Reports 99 (1983) 66.

/2/ BJORKEN J., Phys. Rev. 148 (1966) 1467.

13/ CARLITZ R. and KAUR J., Phys. Rev. Lett. 38 (1977) 673.

14/ BABCOCK J. et al., Phys. Rev. D19 (1979) 1483.

15/ CHIAPPETTA P. and SOFFER J., Phys. Rev. D (to be published) and these proceedings.

/6/ BAUM G. et al., Phys. Rev. Lett. 51 (1983) 1135.

17/ CRAIGIE N. et a1., z. Phys. C12 (1982) 173.

18/ CALlaWAY D. and Ellis S., Phys. Rev. D29 (1984) 567.

19/ LAM C.S. and LI B., Phys. Rev. D25 (1982) 683.

/10/ BIGI I., Nuovo Cimento 41A (1977) 43 and 581.

/11/ DONOGHUE J., Phys. Rev. D17 (1978) 2922;

BARTL A. et a1., Z. Phys. C6 (1980) 335.

/12/ HAGIWARA K. et a1., Phys. Rev. D27 (1983) 84.

/13/ WITTEN E., Nuc1. Phys. B120 (1977) 189; DELDUC F. et al., Nucl. Phys. B174 (1980) 147 and 157.

/14/ GLUCK M. and REYA E., Phys. Rev. D28 (1983) 2749.

/15/ LAYSSAC J. et al., Z. Phys. C25 (1984) 49 and these proceedings; see also: LIU H., Phys. Rev. D29 (1984) 832 .

/16/ DEvoTo A. et al., Phys. Rev, Lett. 43 (1979) 1062.

/17/ FONTANNAZ M. and SCHIFF D., Z. Phys. Cl4 (1982) 151.

/18/ DUKE D. and OWENS J., Phys. Rev. Lett. 44 (1980) 1173.

119/ PETERSSON B. and PIRE B., Phys. Lett. 95B (1980) 119.

/20/ BRODSKY s. et a1., Phys. Lett. 79B (1978) 255.

/21/ ANSELMINO M. and KROLL P., Phys. Rev. D30 (1984) 36.

/22/ FONTANNAZ M. et al., Z. Phys. C8 (1981) 349.

/23/ XU Z., Brown Universtty preprint HET-523 (1984).

124/ RATCLIFFE P., Nucl. Phys. B223 (1983) 45;

KARASINSKI P., Phys. Rev. D29 (1984) 1039.

125/ WATSON A., 2. Phys. C12 (1982) 123; Nuovo Cimento 42A (1984) 661. 